\title{
An-Luo-Hua-Xian Pill Can Improve the Regression of Liver Fibrosis in Chronic Hepatitis B Patients Treated With Entecavir
}

Yi-Qi Liu

Peking University First Hospital

Chi Zhang

Peking University First Hospital

Jia-Wen Li

Peking University First Hospital

Li-Hua Cao

the third Hospital of Qinhuangdao

Zhan-Qing Zhang

Shanghai Public Health Clinical Center

Wei-Feng Zhao

xinxiang medical university third hospital

Qing-Hua Shang

No.88 hospital fo chinese PLA

Da-Zhi Zhang

the second affiliated hospital of chongqing medical university

\section{An-Lin Ma}

China-Japan Friendship Hospital

\section{Qing Xie}

Shanghai Jiao Tong University Medical School Affiliated Ruijin Hospital

\section{Hong-Lian Gui}

Shanghai Jiao Tong University Medical School Affiliated Ruijin Hospital

\section{Guo Zhang}

Peoples Hospital of Guangxi Zhuang Autonomous Region: Nanxishan Hospital of Guangxi Zhuang Autonomous Region

\section{Ying-Xia Liu}

The Third People's Hospital of Shenzhen

\section{Jia Shang}

the peoples hospital of henan province

\section{Shi-Bin Xie}

The Third Affiliated Hospital of Sun Yet-sun University 
Jun Li

Jiangsu Province Hospital and Nanjing Medical University First Affiliated Hospital

\section{Xu-Qing Zhang}

the southwest hospital of army medical university

\section{Zhi-Qiang Zou}

yantai infectious diseases hospital

\section{Yu-Ping Chen}

baoding infectious diseases hoapital

\section{Zong Zhang}

jinan infectious diseases hospital

\section{Ming-Xiang Zhang}

the sixth people's hospital of shenyang

\section{Jun Cheng}

Capital Medical University Affiliated Beijing Ditan Hospital

\section{Fu-Chun Zhang}

Guangzhou Eighth People's Hospital

\section{Li-Hua Huang}

wuxi no 5 people's hospital

\section{Jia-Bin Li}

First Affiliated Hospital of Anhui Medical University

\section{Qing-Hua Meng}

Capital Medical University Youan Hospital

\section{Hai-Bin Yu}

Capital Medical University Youan Hospital

\section{Yu-Qiang Mi}

Tianjin City Second People's Hospital

\section{Yan-Zhong Peng}

Peking University Shenzhen Hospital

\section{Zhi-Jin Wang}

305 hospial of Chinese PLA

\section{Li-Ming Chen}

5th Medical Center of Chinese PLA General Hospital

\section{Fan-Ping Meng}

5th Medical Center of Chinese PLA General Hospital

\section{Wan-Hua Ren}

Shandong Provincial Hospital

\section{Lang Bai}

West China School of Medicine: Sichuan University West China Hospital

\section{Yi-Lan Zeng}


the public hospital center of chengdu

\section{Rong Fan}

Southern Medical University Nanfang Hospital

\section{Xian-Zhi Lou}

Central Hospital affiliated to Shenyang Medical College

\section{Wei-Feng Liang}

First Hospital of Zhejiang Province: Zhejiang University School of Medicine First Affiliated Hospital

Hui Liu

Capital Medical University Youan Hospital

\section{Hui Zhuang}

Peking University Health Science Center

Hong Zhao

Peking University First Hospital

Guiqiang Wang ( $\sim$ john131212@126.com )

Peking University First Hospital https://orcid.org/0000-0002-0317-7536

\section{Research Article}

Keywords: chronic hepatitis B, liver fibrosis, cirrhosis, liver pathology, ALHX pill, ETV, fibrosis regression, LSM, randomized controlled trial, mainland China

Posted Date: January 4th, 2022

DOI: https://doi.org/10.21203/rs.3.rs-1192811/v1

License: (c) (i) This work is licensed under a Creative Commons Attribution 4.0 International License. Read Full License 


\section{Abstract}

Background \& Aims: Chronic hepatitis B (CHB) can cause liver fibrosis and lead to cirrhosis even cancer, antiviral therapy can reverse liver fibrosis with limited effect, thus we aimed to evaluate the effect of AnLuo-Hua-Xian pill (ALHX) on fibrosis regression in CHB patients treated with ETV.

Methods: Treatment naïve patients with CHB from Oct $1^{\text {st }}, 2013$ to Dec $31^{\text {st }}, 2020$ were randomly treated with ETV alone or combined with ALHX (ETV+ALHX). Patients' demographic, laboratory and liver histology data before and after 78 weeks of treatment were collected. Ishak fibrosis score (F) was used and fibrosis regression means $\mathrm{F}$ decreased $\geq 1$ after treatment.

Results: In total, 394 patients with a second liver biopsy after treatment were included in per-protocol population: 132 patients in ETV group and 262 patients in ETV+ALHX group. After 78 weeks of treatment, the fibrosis regression rate in the ETV+ALHX group were significantly higher than ETV group in baseline F $\geq 3$ patients: $124 / 211$ (58.8\%) verse $45 / 98$ (45.9\%), $p=0.035$. The degradation rate of liver stiffness measurement (LSM) is also consistent with it: 154/262 (73.0\%) in ETV+ALHX group and 60/132 (61.2\%) in ETV group, $p=0.037$. Logistic regression analysis showed that combined with ALHX was related to fibrosis regression (OR=1.94, $p=0.018)$, and family history of hepatocellular carcinoma was on the contrary $(\mathrm{OR}=0.41, p=0.031)$.

Conclusions: ETV combined with ALHX can significantly increase liver fibrosis regression in CHB patients.

\section{Introduction}

Chronic hepatitis B virus (HBV) infection is one of the serious public health problems endangering people's health especially in China ${ }^{1}$. Liver fibrosis caused by chronic hepatitis $B(\mathrm{CHB})$ is the key in occurrence of liver cirrhosis and liver cancer. If $\mathrm{CHB}$ patients are not diagnosed and treated in time, most of them will die of decompensated liver cirrhosis or HCC. CHB accounts for $30 \%$ of all liver cirrhosis death and $40 \%$ of HCC death ${ }^{2}$. A large number of research results showed that effective antiviral therapy can help to improve HBV related liver fibrosis and the improvement rate of hepatic fibrosis in newly treated patients with CHB patients treated with entecavir (ETV) for 48 weeks was $32 \%-39 \%{ }^{3}$. Although liver fibrosis has not been improved in more than half of patients, there are still no clearly recommended antifibrosis drugs.

In recent decades, through continuous research and experiments, Traditional Chinese medicine (TCM) has made remarkable progress in the treatment of chronic liver disease, and many drugs for the treatment of liver fibrosis have been approved by the State Drug Administration of China. Studies have shown that TCM alone or combined with anti HBV drugs has a good effect in delaying or reversing liver fibrosis/cirrhosis ${ }^{4,5}$. An-Luo-Hua-Xian pill (ALHX) is a Chinese patent drug containing more than ten kinds of TCMs, such as Rehmannia glutinosa (Di-Huang), Radices pseudo-ginseng (San-Qi) and Leech (Shui- 
Zhi). "Luo" in Chinese means collateral branches of the pathway system that runs the "Qi" and blood of the whole body, "Xian" means fibrosis, "An" means to calm or pacify and "Hua" means to soften or alleviate. ALHX can regulate immunity, improve liver microcirculation, promote hepatocyte injury and repair collagen synthesis and promote collagen degradation ${ }^{6}$. Our previous study already found that in significant fibrosis (fibrosis grade $\geq 3$ ) CHB patients, ETV combined with ALHX can significantly improve the regression rate of liver fibrosis/cirrhosis than ETV alone ${ }^{7}$. But further expand the sample size to confirm the curative effect, form a standardized treatment scheme for reversing liver fibrosis and promote its application is needed.

Thus, based on the previous research, we continued to carry out a prospective randomized controlled trial in order to further clarify the anti-fibrosis effect of ALHX in CHB patients.

\section{Methods}

\subsection{Study design and patients}

This is a multicenter, open-label prospective randomized controlled trial in 33 hospitals in mainland China. Eligible patients consented to participate in the study between Oct $1^{\text {st }}, 2013$ to Dec 31 st, 2020. The inclusion criteria were: 1) 18-70 years old; 2) positive of hepatitis B surface antigen (HBsAg+) $\geq 6$ months or pathologically judged as chronic HBV infection; 3) HBV DNA positive; 4) regular follow-up. Exclusion criteria were: 1 ) other types of viral hepatitis: hepatitis $C$ virus infection (HCV), hepatitis D virus (HDV) or human immunodeficiency virus (HIV) co-infection and other chronic liver diseases (autoimmune hepatitis, drug-induced liver damage, genetic, nonalcoholic fatty liver); 2) decompensated manifestations of liver cirrhosis, including ascites, hepatic encephalopathy, gastrointestinal bleeding, hepatorenal syndrome, spontaneous bacterial peritonitis and other complications of liver cirrhosis or primary liver cancer; 3 ) unstable diabetes, hypertension, thyroid diseases and autoimmune diseases; 4) patients with serious diseases of heart, lung, kidney, brain, blood and other important organs with dysfunction; 5) patients with severe neurological and mental diseases (such as epilepsy, depression, mania, schizophrenia, etc.); 6) pregnant and lactating women.

Patients were randomly assigned to either the ETV+ALHX group or the ETV group, in the ratio of 2:1, by simple randomization with no stratification. Randomized treatment was open-label. Patients were assigned to a serial number by the study coordinator which was linked to a computer-generated randomization list assigning the treatment regimens.

All patients signed informed consent before enrollment. Procedures followed were in accordance with the Helsinki Declaration and was approved by The Ethical Committees of Peking University First Hospital. The complete protocol for the clinical trial has been registered at ClinicalTrials.gov (NCT01962155 and NCT03568578).

\subsection{Liver biopsy and scoring system}


Liver histological specimens (paraffin embedded) by patients who underwent a percutaneous ultrasoundguided liver biopsy will be transported to the central laboratory for unified interpretation, specimens were considered adequate for scoring with a length more than $2.0 \mathrm{~cm}$ and containing at least 11 portal tracts and assessed by two professional liver pathologists from Capital Medical University affiliated Beijing You-An Hospital under double-blind conditions. If the results of the two pathologists are different or the interpretation results have large difference with the local pathology, the results will be determined under the joint discussion of the third pathologist.

The evaluation of liver fibrosis $(F)$ was divided into 0-6 stages and necroinflammatory scores was using modified histological activity index (HAl) grading by Ishak scoring and grading system, $F \geq 3$ was considered significant fibrosis ${ }^{8}$. Fibrosis changes after treatment: regression means $\mathrm{F}$ decreased $\geq 1$ after treatment and progress means $F$ increased at least one stage after treatment. Histological improvement means HAl decreased at least two grade and no progression (increased more than and equal to one stage) in fibrosis after treatment.

\subsection{Endpoints}

The primary endpoint includes liver fibrosis regression and liver stiffness measurement (LSM) degradation. LSM was divided into four levels ${ }^{9,10}$ : $\mathrm{LSM}<7.4,7.4 \leq \mathrm{LSM}<9.0,9.0 \leq \mathrm{LSM}<12.0$ and $\geq 12.0$, "LSM degradation" means grade after treatment decreased at least one than before. "LSM degradation" means that the level after treatment is at least one level lower than that before treatment.

Secondary endpoints included histological improvement and non-invasive fibrosis indexes degradation, biochemical, virological and serological responses. Non-invasive fibrosis indexes: fibrosis-4-score (FIB-4) and aspartate aminotransferase to platelet ratio index (APRI) were calculated by biochemical datas. FIB-4 was divided into three levels 11,12: FIB-4<1.45, FIB-4 $\geq 3.25$ and between them, "FIB-4 degradation" means the same as LSM. APRI was divided into three levels ${ }^{13}$ : APRI $<1.00$, APRI $\geq 2.00$ and between them, "APRI degradation" means the same as LSM too.

Qualtitative detection of HBsAg was performed using available enzyme immunoassays (Roche Diagnostics Co., Penzberg, Germany), HBV DNA was tested by Roche COBAS AmpliPrep/COBAS TaqMan (Roche Co., Penzberg, Germany) with a minimum detection value of $20 \mathrm{IU} / \mathrm{mL}$ and quantification of serum hepatitis B core antibody (qAnti-HBc) was measured by a newly chemiluminescent particle immunoassay (Wantai Co., Xiamen, China), which were all tested in the central laboratory in Peking University First Hospital. Biochemical data and transient elastography results (liver stiffness measurement, LSM) were collected in local center.

\subsection{Statistical analysis}

Quantitative variables were expressed as the median (lower and upper quartiles) or mean \pm standard deviation. Categorical variables were demonstrated with number and percentage. The t-test or KruskalWallis analysis were used to compare continuous variables, and Chi-square or Fisher's exact tests were used to categorical variables. Logistic regression was used to analyze the related factors of fibrosis 
regression. The $p<0.05$ was considered to be statistically significant. Statistical analyses were performed using SPSS 24.0 (SPSS, Chicago, IL).

All authors had access to the study data and reviewed and approved the final manuscript.

\section{Results}

\subsection{Study population}

From Oct $1^{\text {st }}, 2013$ to Dec 31st, 2020, a total of $1328 \mathrm{HBsAg}(+)$ patients were assessed, $780 \mathrm{CHB}$ patients with liver biopsy were randomized. A total of 394 patients with a second liver biopsy after treatment were finally enrolled in the per-protocol population: 132 patients in the ETV group and 262 patients in the ETV+ALHX group. The enrollment protocol of patients was showed in Figure 1. Both groups were mainly male patients and the average age was about 40 years old, body mass index (BMI) is about $23-24 \mathrm{~kg} / \mathrm{m}^{2}$. There was no significant difference between two groups in the baseline demographic and clinical characteristics except HAI grade. The baseline characteristics of the patients were presented in Table 1. Baseline characteristics of patients with significant fibrosis were presented in Supplement Table 1. 
Table 1

Baseline demographic and clinical characteristics.

\begin{tabular}{|c|c|c|c|}
\hline & ETV & ETV+ALHX & $p$-value \\
\hline $\mathrm{N}$ & 132 & 262 & \\
\hline Age, year & $40.68 \pm 11.58$ & $40.92 \pm 10.07$ & 0.831 \\
\hline$<40, \mathrm{n}(\%)$ & $63(47.7)$ & $125(47.7)$ & \\
\hline$\geq 40, \mathrm{n}(\%)$ & $69(52.3)$ & $137(52.3)$ & \\
\hline Male sex, $\mathrm{n}(\%)$ & 104(78.8) & $190(72.5)$ & 0.177 \\
\hline $\mathrm{BMI}\left(\mathrm{kg} / \mathrm{m}^{2}\right)$ & $23.52 \pm 3.03$ & $24.00 \pm 3.27$ & 0.163 \\
\hline Family history of HBV infection, $\mathrm{n}(\%)$ & $57(43.2)$ & $131(50.4)$ & 0.177 \\
\hline Family history of HCC, n(\%) & $17(12.9)$ & $31(11.9)$ & 0.775 \\
\hline History of drinking, n(\%) & $4(3.0)$ & $5(1.9)$ & 0.491 \\
\hline History of smoking, $\mathrm{n}(\%)$ & 26(19.7) & $65(24.9)$ & 0.248 \\
\hline HBsAg positive duration, year & $10(3,20)$ & $11(4,20)$ & 0.270 \\
\hline WBC $\left(\times 10^{\wedge} 9 / L\right)$ & $5.49 \pm 1.5,, 5.36$ & $5.33 \pm 1.4,, 5.11$ & 0.295 \\
\hline LY\% & $34.99 \pm 8.6,35.50$ & $35.13 \pm 8.8,35.10$ & 0.879 \\
\hline $\mathrm{HGB}(\mathrm{g} / \mathrm{L})$ & $146.77 \pm 16.9,, 150$ & $144.74 \pm 16 .,, 147$ & 0.259 \\
\hline $\operatorname{PLT}\left(\times 10^{\wedge} 9 / \mathrm{L}\right)$ & $161.08 \pm 50.1,, 158.50$ & $156.87 \pm 52.6,, 153.00$ & 0.447 \\
\hline $\operatorname{ALT}(\mathrm{U} / \mathrm{L})$ & $57.30(37.71,106.00)$ & $56.00(34.00,105.71)$ & 0.173 \\
\hline AST(U/L) & $39.38(28.00,67.95)$ & $41.00(29.00,67.95)$ & 0.053 \\
\hline $\operatorname{ALP}(U / L)$ & $86.00(73.80,112.00)$ & $82.46(67.00,108.64)$ & 0.516 \\
\hline GGT(U/L) & $37.65(25.10,73.80)$ & $41.33(25.00,78.15)$ & 0.872 \\
\hline $\operatorname{ALB}(\mathrm{g} / \mathrm{L})$ & $43.37 \pm 4.5,43.05$ & $42.60 \pm 4.8,43.00$ & 0.650 \\
\hline TBiL $(\mu \mathrm{mol} / \mathrm{L})$ & $15.40(10.80,19.60)$ & $14.60(11.70,20.00)$ & 0.251 \\
\hline $\operatorname{DBiL}(\mu \mathrm{mol} / \mathrm{L})$ & $4.95(3.35,6.90)$ & $4.60(3.40,7.50)$ & 0.123 \\
\hline
\end{tabular}

All values shown are based on available data, and numeric data are represented as (mean $\pm S D$, median) or median(range); Abbreviations: HBV, hepatitis B virus; HCC, hepatocellular carcinoma; BMI, body mass index; WBC, white blood cell; LY\%, percentage of lymphocyte; HGB, hemoglobin; PLT, platelet; ALT, alanine aminotransferase; AST, aspartate aminotransferase; ALP, alkaline phosphatase; GGT, glutamyl transferase; ALB, albumin; TBil, total bilirubin; DBil, direct bilirubin; $\mathrm{Cr}$, creatinine; TCHO, total cholesterol; TG, triglyceride; AFP, alpha fetoprotein; INR, international normalized ratio; LSM, liver stiffness measurement; qAnti-HBc, quantitative anti-hepatitis B core antigen; HAl, liver histologic activity scores of inflammatory; F, liver fibrosis score. 


\begin{tabular}{|c|c|c|c|}
\hline & ETV & ETV+ALHX & $p$-value \\
\hline $\operatorname{Cr}(\mu \mathrm{mol} / \mathrm{L})$ & $66.77 \pm 13.0,66.00$ & $67.84 \pm 14.5,66.90$ & 0.960 \\
\hline $\mathrm{TCHO}(\mathrm{mmol} / \mathrm{L})$ & $4.26 \pm 0.8,4.09$ & $4.40 \pm 0.8,4.42$ & 0.122 \\
\hline TG (mmol/L) & $1.10 \pm 0.5,0.95$ & $1.15 \pm 0.5,1.02$ & 0.411 \\
\hline AFP(ng/mL) & $4.59(2.82,7.59)$ & $4.60(2.75,15.32)$ & 0.815 \\
\hline INR & $1.09 \pm 0.1,1.09$ & $1.09 \pm 0.1,1.08$ & 0.975 \\
\hline HBV DNA $\left(\log _{10} \mathrm{IU} / \mathrm{ml}\right)$ & $5.78 \pm 1.7,6.01$ & $5.63 \pm 1.9,, 5.75$ & 0.470 \\
\hline $\mathrm{HBsAg}\left(\log _{10} \mathrm{IU} / \mathrm{ml}\right)$ & $3.32 \pm 0.7,, 3.41$ & $3.36 \pm 0.7,3.39$ & 0.589 \\
\hline $\mathrm{HBeAg}(+), \mathrm{n}(\%)$ & $84(63.6)$ & $138(52.7)$ & 0.053 \\
\hline qAnti-HBc( $\left.\log _{10} \mathrm{IU} / \mathrm{ml}\right)$ & $3.73 \pm 0.7,3.75$ & $3.85 \pm 0.7,3.91$ & 0.149 \\
\hline LSM (kPa) & $10.45(7.80,16.30)$ & $11.90(8.30,18.00)$ & 0.117 \\
\hline HAl & & & 0.025 \\
\hline$\leq 4, \mathrm{n}(\%)$ & $41(31.1)$ & $68(26.0)$ & \\
\hline $5-6, n(\%)$ & $50(37.9)$ & $72(27.5)$ & \\
\hline $7-9, n(\%)$ & $30(22.7)$ & 83(31.7) & \\
\hline$\geq 10, n(\%)$ & $11(8.3)$ & $39(14.9)$ & \\
\hline $\mathrm{F}$ & & & 0.306 \\
\hline$<3, \mathrm{n}(\%)$ & $34(25.8)$ & $51(19.5)$ & \\
\hline $3-4, n(\%)$ & $68(51.5)$ & $153(58.4)$ & \\
\hline $5-6, n(\%)$ & $30(22.7)$ & $58(22.1)$ & \\
\hline \multicolumn{4}{|c|}{$\begin{array}{l}\text { All values shown are based on available data, and numeric data are represented as (mean } \pm \text { SD, } \\
\text { median) or median(range); Abbreviations: HBV, hepatitis B virus; HCC, hepatocellular carcinoma; BMI, } \\
\text { body mass index; WBC, white blood cell; LY\%, percentage of lymphocyte; HGB, hemoglobin; PLT, } \\
\text { platelet; ALT, alanine aminotransferase; AST, aspartate aminotransferase; ALP, alkaline phosphatase; } \\
\text { GGT, glutamyl transferase; ALB, albumin; TBil, total bilirubin; DBil, direct bilirubin; Cr, creatinine; TCHO, } \\
\text { total cholesterol; TG, triglyceride; AFP, alpha fetoprotein; INR, international normalized ratio; LSM, liver } \\
\text { stiffness measurement; qAnti-HBc, quantitative anti-hepatitis B core antigen; HAl, liver histologic } \\
\text { activity scores of inflammatory; F, liver fibrosis score. }\end{array}$} \\
\hline
\end{tabular}

\subsection{Endpoints}

\subsubsection{Primary endpoint}


Patients with baseline $F \geq 3$ in the ETV+ALHX group has a significantly higher rate $(124 / 211,58.8 \%)$ of fibrosis regression than in the ETV group $(45 / 98,45.9 \%)$ after $78 w$ treatment, $p=0.035$. (Table 2$)$. All patients in ETV+ALHX group also has a higher fibrosis regression rate than ETV group but without significant difference: $130 / 262(49.6 \%)$ and $53 / 132(40.2 \%), p=0.075$. (Table 2.). The distribution of $F$ stage at baseline and after $78 \mathrm{w}$ treatment for both groups were shown in Figure 2. Different baseline in fibrosis changes after treatment were showed in supplement Table 2. 
Table 2

Treatment efficacy of the ETV group and ETV+ALHX group.

\begin{tabular}{|c|c|c|c|}
\hline & ETV & ETV+ALHX & p-value \\
\hline All patients, $\mathrm{n}$ & 132 & 262 & \\
\hline ALT normalization, n(\%) & $83 / 90(92.2)$ & $159 / 178(88.8)$ & 0.449 \\
\hline HBV DNA decreased $\geq 2 \times \lg , \mathrm{n}(\%)$ & 118(89.4) & 238(90.8) & 0.646 \\
\hline HBV DNA $\leq 20 \mathrm{IU} / \mathrm{ml}, \mathrm{n}(\%)$ & $100(75.8)$ & 203(77.5) & 0.702 \\
\hline HBeAg clearance, n(\%) & 26(19.7) & $44(16.9)$ & 0.498 \\
\hline HBeAg seroconversion, $n(\%)$ & $9(6.8)$ & 11(13.3) & 0.271 \\
\hline Histological improvement ${ }^{1}, \mathrm{n}(\%)$ & $61(46.2)$ & $145(55.3)$ & 0.087 \\
\hline HAl decreased $\geq 2, n(\%)$ & $74(56.1)$ & $173(66.0)$ & 0.053 \\
\hline \multicolumn{4}{|l|}{ Fibrosis changes $^{2}$ : } \\
\hline Regression, $\mathrm{n}(\%)$ & $53(40.2)$ & $130(49.6)$ & 0.075 \\
\hline Progression, n(\%) & $30(25.1)$ & $45(17.2)$ & 0.185 \\
\hline LSM degradation ${ }^{1}, \mathrm{n}(\%)$ & $85(64.4)$ & $179(68.3)$ & 0.434 \\
\hline FIB-4 degradation ${ }^{2}, \mathrm{n}(\%)$ & $47(35.6)$ & $96(36.6)$ & 0.840 \\
\hline APRI degradation ${ }^{3}, \mathrm{n}(\%)$ & $47(35.6)$ & $107(40.8)$ & 0.315 \\
\hline $\mathrm{F} \geq 3$ patients, $\mathrm{n}$ & 98 & 211 & \\
\hline ALT normalization, n(\%) & $65 / 70(92.9)$ & $126 / 144(87.5)$ & 0.235 \\
\hline HBV DNA decreased $\geq 2 \log _{10}, n(\%)$ & $90(91.8)$ & $191(90.5)$ & 0.708 \\
\hline $\mathrm{HBV}$ DNA $\leq 20 \mathrm{IU} / \mathrm{ml}$ & $75(76.5)$ & $166(78.7)$ & 0.672 \\
\hline HBeAg clearance, n(\%) & 14(14.3) & $33(15.8)$ & 0.733 \\
\hline HBeAg seroconversion, n(\%) & $1(1.0)$ & $8(3.8)$ & 0.174 \\
\hline Histological improvement ${ }^{1}, \mathrm{n}(\%)$ & $48(49.0)$ & $123(58.3)$ & 0.125 \\
\hline HAl decreased $\geq 2, n(\%)$ & $52(53.1)$ & $142(67.3)$ & 0.016 \\
\hline \multicolumn{4}{|l|}{ Fibrosis changes 2 : } \\
\hline Regression, $\mathrm{n}(\%)$ & $45(45.9)$ & $124(58.8)$ & 0.035 \\
\hline Progression, n(\%) & 13(13.3) & 29(13.7) & 0.909 \\
\hline
\end{tabular}




\begin{tabular}{|llll|}
\hline & ETV & ETV+ALHX & p-value \\
\hline LSM degradation ${ }^{1}, \mathrm{n}(\%)$ & $60(61.2)$ & $154(73.0)$ & 0.037 \\
\hline FIB-4 degradation ${ }^{2}, \mathrm{n}(\%)$ & $33(33.7)$ & $77(36.5)$ & 0.630 \\
\hline APRI degradation ${ }^{3}, \mathrm{n}(\%)$ & $37(37.8)$ & $88(41.7)$ & 0.510 \\
\hline
\end{tabular}

We also measured fibrosis regression in LSM, APRI and FIB-4. Patients with baseline $F \geq 3$, LSM degradation rate in ETV+ALHX group (73.0\%) was much higher than ETV group (61.2\%), $p=0.037$. FIB-4 and APRI in the two groups before and after treatment were also showed in Table 2. The distribution of LSM, APRI and FIB-4 at baseline and after treatment and changes of them between the two groups before and after treatment were all showed in Figure 3.

\subsubsection{Secondary endpoint}

The normalization rate of ALT, the rate of HBV DNA decreased $\geq$ twice the log value, the rate of HBV DNA $\leq 20 \mathrm{lU} / \mathrm{ml}$, HBeAg clearance rate and HBeAg seroconversion rate in ETV+ALHX group and ETV group were all without significant difference. (Table 2)

But the rate of HAI decreased $\geq 2$ grade (in baseline $\mathrm{F} \geq 3$ patients), ETV+ALHX group was significantly higher than ETV group $(67.3 \%$ vs $53.1 \%, p=0.016)$. Histological improvement rate in ETV+ALHX group also has a higher trend both in all patient ( $55.3 \%$ vs $46.2 \%$ ) and $\mathrm{F} \geq 3$ patients (58.3\% vs $49.0 \%$ ). (Table 2 ).

\subsection{Factors related to fibrosis regression}

We divided significant fibrosis patients (baseline $\mathrm{F} \geq 3$ ) into regression group and not regression group (the stage of baseline $F$ is equal to or increased after treatment) according to the treatment outcome. And we found that ETV combined with ALHX had a higher rate in fibrosis regression ( $73.4 \%$ vs $62.1 \%$, $p=0.035)$ and family history of HCC made patients more difficult to reach fibrosis regression $(7.7 \% \mathrm{vs}$ $17.1 \%, p=0.026)$. Variables with $p<0.2$ in univariate analysis were selected into logistic regression for multivariate analysis, we found that combined with ALHX, no family history of HCC, high PLT and low INR made a significant difference related to fibrosis regression. Detailed results were showed in Table 3. 
Table 3

Analysis of related factors of fibrosis regression (in $\mathrm{F} \geq 3$ patients).

\begin{tabular}{|c|c|c|c|c|c|c|}
\hline & Regression & Not regression & uni & $p^{1}$ & multi & $p^{2}$ \\
\hline$N$ & 169 & 140 & & & & \\
\hline ETV+ALHX group & 124(73.4) & $87(62.1)$ & 4.46 & 0.035 & $\begin{array}{l}1.94(1.12, \\
3.37)\end{array}$ & 0.018 \\
\hline Age(years) & $41.12 \pm 10.51$ & $42.34 \pm 10.72$ & -1.00 & 0.316 & & \\
\hline$<40, \mathrm{n}(\%)$ & $79(46.7)$ & $58(41.4)$ & 0.88 & 0.349 & & \\
\hline Male sex, $\mathrm{n}(\%)$ & 121(71.6) & 102(72.9) & 0.06 & 0.81 & & \\
\hline $\begin{array}{l}\text { Family history of HBV } \\
\text { infection, } \mathrm{n}(\%)\end{array}$ & $81 / 167(48.5)$ & $72(51.4)$ & 0.26 & 0.610 & & \\
\hline $\begin{array}{l}\text { Family history of HCC, } \\
\mathrm{n}(\%)\end{array}$ & 13/168(7.7) & $24(17.1)$ & 6.39 & 0.011 & $\begin{array}{l}0.41(0.18 \\
0,92)\end{array}$ & 0.031 \\
\hline History of drinking, $\mathrm{n}(\%)$ & $5(3.0)$ & $4(2.9)$ & 0.00 & 0.958 & & \\
\hline $\begin{array}{l}\text { HBsAg positive } \\
\text { duration, year }\end{array}$ & $11(4,20)$ & $10(2,20)$ & -0.26 & 0.794 & & \\
\hline $\mathrm{BMI}\left(\mathrm{kg} / \mathrm{m}^{2}\right)$ & $\begin{array}{l}23.98 \pm 3.4 \\
, 23.62\end{array}$ & $\begin{array}{l}24.22 \pm 3.0 \\
, 24.22\end{array}$ & -0.64 & 0.524 & & \\
\hline WBC $\left(\times 10^{\wedge} 9 / L\right)$ & $5.47 \pm 1.5,, 5.30$ & $5.32 \pm 1.5,, 5.18$ & 0.77 & 0.443 & & \\
\hline LY\% & $\begin{array}{l}34.93 \pm 7.8 \\
, 34.6\end{array}$ & $\begin{array}{l}34.83 \pm 9.6 \\
, 35.9\end{array}$ & 0.11 & 0.918 & & \\
\hline $\operatorname{HGB}(g / L)$ & $\begin{array}{l}143.91 \pm 17.3, \\
, 147\end{array}$ & $\begin{array}{l}146.22 \pm 16.7 \\
, 148\end{array}$ & -1.19 & 0.237 & & \\
\hline $\operatorname{PLT}\left(\times 10^{\wedge} 9 / \mathrm{L}\right)$ & $\begin{array}{l}157.57 \pm 52.2 \\
, 155.00\end{array}$ & $\begin{array}{l}144.86 \pm 46.0 \\
, 143.00\end{array}$ & 2.25 & 0.026 & $\begin{array}{l}1.00(1.00, \\
1.01)\end{array}$ & 0.012 \\
\hline $\operatorname{ALT}(\mathrm{U} / \mathrm{L})$ & $\begin{array}{l}56.00(38.00 \\
101.28)\end{array}$ & $\begin{array}{l}56.00(35.00 \\
101.28)\end{array}$ & 1.16 & 0.265 & & \\
\hline AST(U/L) & $\begin{array}{l}41.00(28.00 \\
70.00)\end{array}$ & $\begin{array}{l}41.00(30.09 \\
69.26)\end{array}$ & 1.11 & 0.239 & & \\
\hline $\operatorname{ALP}(U / L)$ & $\begin{array}{l}\text { 87.15(71.05, } \\
109.22)\end{array}$ & $\begin{array}{l}\text { 89.32(71.28, } \\
114.91)\end{array}$ & -0.11 & 0.914 & & \\
\hline GGT(U/L) & $\begin{array}{l}46.00(28.50 \\
80.00)\end{array}$ & $\begin{array}{l}42.78(24.91 \\
83.81)\end{array}$ & -0.16 & 0.870 & & \\
\hline $\operatorname{ALB}(\mathrm{g} / \mathrm{L})$ & $\begin{array}{l}42.46 \pm 4.9 \\
43.00\end{array}$ & $\begin{array}{l}41.68 \pm 4.8 \\
, 42.00\end{array}$ & 1.41 & 0.161 & & 0.943 \\
\hline $\operatorname{TBil}(\mu \mathrm{mol} / \mathrm{L})$ & $\begin{array}{l}\text { 14.70(11.40, } \\
20.80)\end{array}$ & $\begin{array}{l}\text { 15.90(11.45, } \\
20.30)\end{array}$ & 1.27 & 0.177 & & 0.217 \\
\hline
\end{tabular}




\begin{tabular}{|c|c|c|c|c|c|c|}
\hline & Regression & Not regression & uni & $p^{1}$ & multi & $p^{2}$ \\
\hline $\operatorname{DBil}(\mu \mathrm{mol} / \mathrm{L})$ & $\begin{array}{l}4.70(3.21 \\
7.50)\end{array}$ & $\begin{array}{l}4.70(3.40 \\
7.65)\end{array}$ & 1.71 & 0.065 & & 0.077 \\
\hline $\operatorname{Cr}(\mu \mathrm{mol} / \mathrm{L})$ & $\begin{array}{l}66.97 \pm 14.1 \\
, 66.00\end{array}$ & $\begin{array}{l}65.81 \pm 12.9 \\
, 65.40\end{array}$ & 0.75 & 0.457 & & \\
\hline $\mathrm{TCHO}(\mathrm{mmol} / \mathrm{L})$ & $4.42 \pm 0.9,4.31$ & $4.23 \pm 0.7,4.20$ & 1.85 & 0.060 & & 0.688 \\
\hline $\mathrm{TG}(\mathrm{mmol} / \mathrm{L})$ & $1.19 \pm 0.6,1.01$ & $1.08 \pm 0.5,1.02$ & 1.59 & 0.113 & & 0.798 \\
\hline $\operatorname{AFP}(\mathrm{ng} / \mathrm{mL})$ & $\begin{array}{l}4.53(2.94 \\
12.98)\end{array}$ & $\begin{array}{l}5.00(3.10 \\
16.45)\end{array}$ & -0.08 & 0.940 & & \\
\hline INR & $1.10 \pm 0.1,1.08$ & $1.11 \pm 0.1,, 1.10$ & -1.43 & 0.154 & $\begin{array}{l}0.10(0.01 \\
0.87)\end{array}$ & 0.037 \\
\hline $\mathrm{HBV}$ DNA( $\left.\log _{10} \mathrm{IU} / \mathrm{ml}\right)$ & $5.62 \pm 1.9,, 5.68$ & $5.62 \pm 1.7,, 5.83$ & -0.26 & 0.797 & & \\
\hline $\mathrm{HBsAg}\left(\log _{10} \mathrm{IU} / \mathrm{ml}\right)$ & $3.32 \pm 0.6,3.38$ & $3.22 \pm 0.6,3.31$ & 1.32 & 0.189 & & 0.092 \\
\hline $\mathrm{HBeAg}(+), \mathrm{n}(\%)$ & 93/167(55.7) & $80 / 136(58.8)$ & 0.30 & 0.583 & & \\
\hline qAnti-HBc $\left(\log _{10} \mathrm{IU} / \mathrm{ml}\right)$ & $3.88 \pm 0.6,3.90$ & $3.81 \pm 0.8,3.82$ & 0.75 & 0.448 & & \\
\hline LSM (kPa) & $\begin{array}{l}11.90(8.50 \\
18.55)\end{array}$ & $\begin{array}{l}\text { 13.45(8.90, } \\
19.90)\end{array}$ & -0.64 & 0.520 & & \\
\hline HAl & & & 2.42 & 0.490 & & \\
\hline$\leq 4, \mathrm{n}(\%)$ & $38(22.5)$ & $42(30.0)$ & & & & \\
\hline $5-6, n(\%)$ & $53(31.4)$ & $39(27.9)$ & & & & \\
\hline $7-9, n(\%)$ & $49(29.0)$ & $39(27.9)$ & & & & \\
\hline$\geq 10, n(\%)$ & $29(17.2)$ & $20(14.3)$ & & & & \\
\hline $\mathrm{F}$ & & & 30.67 & 0.000 & $\begin{array}{l}2.49(1.81, \\
3.42)\end{array}$ & 0.000 \\
\hline $3-4$ & $99(58.6)$ & 122(87.1) & & & & \\
\hline $5-6$ & $70(41.4)$ & 18(12.9) & & & & \\
\hline
\end{tabular}

\subsection{Safety}

There were two patients in ETV+ALHX group had mild diarrhea, but after evaluated by the attending physician, symptomatic treatment was given and they all relieved soon, so the dosage was not reduced. No noticeable severe adverse effects related to the drug use were observed during the study for both groups. 
In addition, two patients in ETV+ALHX group withdrew from the study due to liver cancer, but they were not related to the experimental drug after evaluation: one is 57 -year-old with a family history of HCC and one is 33-year-old with a known HBsAg positive time of 29 years.

\section{Discussion}

In this study, we found that combined with ALHX has a significant efficacy on the regression of liver fibrosis in CHB patients using ETV, especially in baseline $\mathrm{F} \geq 3$ patients $(p=0.035)$, using transient elastography to estimate liver fibrosis, the LSM degradation rate in ETV+ALHX group was significantly higher than the ETV group ( $p=0.037)$ in baseline $\mathrm{F} \geq 3$ patients, non-invasive fibrosis indexes such as FIB-4 and APRI, the degradation rates in ETV+ALHX group were also higher, although without statistical difference.

Moreover, we calculated that in significant fibrosis patients (baseline $F=3-4$ ), ETV+ALHX group had a higher rate of decreased to less than $3(<3)$ after treatment than ETV group ( $30.1 \%$ vs $19.1 \%, p=0.090)$, and in cirrhosis patients (baseline $\mathrm{F}=5-6)$, the rate were $17.2 \%$ and $3.3 \%(p=0.089)$. And the rate of cirrhosis regression to less than $5(<5)$ after treatment in the two groups were $75.9 \%$ and $63.3 \%$ $(p=0.217)$. This tendency is consistent with other studies such as Kong et al. ${ }^{14}$ found that ETV combined with ALHX can significantly increase the survival rate of decompensated hepatitis B patients after 5-year $(67.85 \%$ vs $26.19 \%, p<0.01)$. Animal model studies have proved that ALHX does play a positive role in anti-fibrosis: Lu. et al. ${ }^{15}$ showed it can reverse carbon tetrachloride (CCl4) induced liver fibrosis in rats, and its mechanism may be through affecting transforming growth factor $\beta-1$ (TGF $\beta-1$ ), which inhibits the activation of hepatic stellate cells (HSC) and plays an anti-fibrosis role. Wang. et al. ${ }^{16}$ found that the antifibrosis effect of ALHX may occur through the upregulation of peroxisome proliferator-activated receptorgamma (PPARY) expression and down regulation of nuclear factor-kappa B (NF-KB) / Inhibitor a of NF-KB $(\mathrm{IKBa})$ signaling pathway. PPARy plays an important role in adipocyte differentiation and lipid metabolism. Its antifibrotic effect may play a role by regulating the adipogenic phenotype of HSC. ${ }^{17} \mathrm{NF}-$ $\mathrm{KB}$ acts as a key regulator of inflammation, NF-KB transcriptional activity increased and maintained at a high level when HSC is activated ${ }^{17}$. However, more clinical studies based on a larger population and more in-depth mechanism studies are still needed to clarify its anti-fibrosis effect.

Other Chinese patent drugs such as Biejia-Ruangan (BR) and Fu-Zheng-Hua-Yu (FZHY) also have an antifibrosis effect in clinical therapy. Rong et al. ${ }^{18}$ enrolled $1000 \mathrm{CHB}$ patients (705 with second biopsy), the rate of fibrosis regression after 72 week treatment was significantly higher in ETV+BR group than ETV group ( $40 \%$ versus $31.8 \%, p=0.0069)$. Huang. et al. ${ }^{19}$ found that BR inhibited hepatic collagen deposition and improved liver injury in $\mathrm{CCl} 4$ induced hepatic fibrosis rats, which might also be associated with down regulation of TGF- $\beta$-Smad pathway. As early as 2005, a multicenter clinical trial confirmed the anti fibrosis effect and safety of FZHY in CHB patients ${ }^{20}$. Gui. et al ${ }^{21}$ carried out a single center clinical study (46 CHB patients with second liver biopsy) showed that ETV+ FZHY had significantly higher rate of fibrosis regression ( $82 \%$ vs $54 \%, p<0.05)$. In mice with fibrosis induced by carbon $(\mathrm{CCl} 4)$ and 
dimethylnitrosamine (DMN), it was observed that FZHY could not only have the effect of anti-fibrosis, but also improve $\mathrm{CCl} 4$ and $\mathrm{DMN}$ induced sinus capillary formation, angiogenesis and angiogenesis related factors' expression ${ }^{22}$. Hepatic sinusoidal capillarization and angiogenesis in the fibrous septum connecting the portal vein and the central hepatic vein are two key events leading to liver cirrhosis. ${ }^{23}$. Further controlled studies are needed to compare the efficacy of these TCMs.

Loomba ${ }^{24}$ et al. found that family history of HCC multiplies the risk of HCC at HBV infection: the cumulative risk was $15.8 \%$ vs $7.5 \%$ ( $p<0.001)$ with or without family history of HCC. And our study suggests that it affects the regression of liver fibrosis, so it may lead to the progression of fibrosis and eventually develop into liver cirrhosis and HCC. Therefore, in guidelines for the diagnosis and treatment of $\mathrm{CHB}$, it is suggested that patients with a family history of HCC should start antiviral treatment. ${ }^{3,25}$. Univariate and multivariate analysis in our study also showed that in significant fibrosis patients, there was a negative correlation between histological fibrosis regression and a family history of HCC. As we know HBV has at least nine different genotypes and genotypes $B$ and $C$ are the most prevalent in China and genotype $\mathrm{C}$ is associated with earlier progression to $\mathrm{HCC}^{26}$. Our research further supports the recommendations of the guidelines and the anti-fibrosis combined with antiviral treatment of these $\mathrm{CHB}$ patients should start it as soon as possible. However, relationship between family history of HCC and fibrosis not to regress or the incidence of HCC still need further mechanism research to clarify.

We have several limitations. Firstly, only nearly half of patients with first liver biopsy did a second liver biopsy after treatment, although we tried our best to communicate with patients, but partly because of the COVID-19, some patients just couldn't return to hospital intime for follow-up. Secondly, ALHX is a patent Chinese drug containing more than ten kinds of TCM, we have not studied the anti- fibrosis effect of single component in depth. Our research showed that ALHX combined with antiviral therapy can significantly reverse liver fibrosis, but its specific anti-fibrosis components need to be studied. Thirdly, our study only observed the results of $78 \mathrm{w}$ of treatment, we still need a longer follow-up to observe and we are working on it.

\section{Conclusion}

In CHB patients using ETV, combined with ALHX can increase liver fibrosis regression rate after $78 \mathrm{w}$ treatment in baseline $\mathrm{F} \geq 3$ patients, whether from the perspective of invasive liver biopsy or non-invasive such as LSM, etc. Logistic regression analysis also proved that ALHX is associated with the regression outcome while family history of HCC is opposite. So, in significant fibrosis patients, we suggest that antiviral therapy should be combined with ALHX as soon as possible.

\section{Declarations}

\section{Declaration of Competing Interest}


The authors declare that they have no known competing financial interests or personal relationships that could have appeared to influence the work reported in this paper.

\section{Compliance with Ethical Requirements}

All procedures followed were in accordance with the ethical standards of the responsible committee on human experimentation and with the Helsinki Declaration. Informed consent was obtained from all patients for being included in the study.

\section{Acknowledgments}

We thank all the patients who participated in this study, as well as all the researchers in local centers. In addition, we thank Cosunter Pharmaceutical Technology and Sunlon Pharmaceutical Technology for providing free drugs, Ashermed Pharmaceutical Technology Co.Ltd for its participation in data checking and collation.

\section{ClinicalTrials.gov number}

NCT01962155 and NCT03568578.

\section{Author contributions}

Conceptualization: Yi-Qi Liu, Hong Zhao, Gui-Qiang Wang; Methodology: Yi-Qi Liu, Chi Zhang, Jia-Wen Li; Formal analysis and investigation: Yi-Qi Liu, Chi Zhang, Jia-Wen Li; Writing - original draft preparation: YiQi Liu; Writing - review and editing: Hui Zhuang, Hong Zhao, Gui-Qiang Wang; Funding acquisition: GuiQiang Wang; Material preparation and data collection: Li-Hua Cao, Zhan-Qing Zhang, Wei-Feng Zhao, Qing-Hua Shang, Da-Zhi Zhang, An-Lin Ma, Qing Xie, Hong-Lian Gui, Guo Zhang, Ying-Xia Liu, Jia Shang, Shi-Bin Xie, Jun Li, Xu-Qing Zhang, Zhi-Qiang Zou, Yu-Ping Chen, Zong Zhang, Ming-Xiang Zhang, Jun Cheng, Fu-Chun Zhang, Li-Hua Huang, Jia-Bin Li, Qing-Hua Meng, Hai-Bin Yu, Yu-Qiang Mi, Yan-Zhong Peng, Zhi-Jin Wang, Li-Ming Chen, Fan-Ping Meng, Wan-Hua Ren, Lang Bai, Yi-Lan Zeng, Rong Fan, XianZhi Lou, Wei-Feng Liang, Hui Liu; Supervision: Hong Zhao, Gui-Qiang Wang.

\section{Funding}

This study was supported by China Mega-Project for Infectious Diseases (grant numbers 2013ZX10002005 and 2017ZX10203202)

\section{References}

1. Collaborators GCoD. Global, regional, and national age-sex-specific mortality for 282 causes of death in 195 countries and territories, 1980-2017: a systematic analysis for the Global Burden of Disease Study 2017. Lancet. Nov 10 2018;392(10159):1736-1788. doi:10.1016/s0140-6736(18)32203-7 
2. Vittal A, Ghany MG. WHO Guidelines for Prevention, Care and Treatment of Individuals Infected with HBV: A US Perspective. Clin Liver Dis. Aug 2019;23(3):417-432. doi:10.1016/j.cld.2019.04.008

3. Terrault NA, Lok ASF, McMahon BJ, et al. Update on prevention, diagnosis, and treatment of chronic hepatitis B: AASLD 2018 hepatitis B guidance. Hepatology. Apr 2018;67(4):1560-1599. doi:10.1002/hep.29800

4. Cheng M, Feng X, Wang L, Yang Y, Ma L, Wang B. Nucleoside analogs assisted with Chinese compound prescription in treating hepatic fibrosis of chronic hepatitis B patients: A protocol of systematic review and meta-analysis. Medicine (Baltimore). Jul 2 2020;99(27):e21032. doi:10.1097/md.0000000000021032

5. Li H. Advances in anti hepatic fibrotic therapy with Traditional Chinese Medicine herbal formula. $J$ Ethnopharmacol. Apr 6 2020;251:112442. doi:10.1016/j.jep.2019.112442

6. Huang J, Huang $\mathrm{H}$, Jiao $\mathrm{Y}$, et al. Effect of anluohuaxian tablet combined with gamma-IFN on schistosomal liver fibrosis. J Huazhong Univ Sci Technolog Med Sci. Feb 2009;29(1):53-8. doi:10.1007/s11596-009-0111-7

7. Liang. M, Wanna. Y, Xiaoqin. D, et al. Combined anluohuaxianwan and entecavir treatment significantly improve the improvement rate of liver fibrosis in patients with chronic hepatitis $B$ virus infection. Chin J Hepatol. 2019;27(7):521-526. doi:10.3760/cma.j.issn.1007-3418.2019.07.009

8. Ishak K, Baptista A, Bianchi L, et al. Histological grading and staging of chronic hepatitis. J Hepatol. Jun 1995;22(6):696-9. doi:10.1016/0168-8278(95)80226-6

9. EASL-ALEH Clinical Practice Guidelines: Non-invasive tests for evaluation of liver disease severity and prognosis. J Hepatol. Jul 2015;63(1):237-64. doi:10.1016/j.jhep.2015.04.006

10. Control CFfHPa. Consensus on clinical application of transient elastography detecting liver fibrosis: a 2018 update. Chin J Hepatol. 2019;27(3):182-191. doi:10.3760/cma.j.issn.1007-3418.2019.03.004

11. Kim WR, Berge T, Asselah T, et al. Evaluation of APRI and FIB-4 scoring systems for non-invasive assessment of hepatic fibrosis in chronic hepatitis B patients. Journal of Hepatology. Apr 2016;64(4):773-780. doi:10.1016/j.jhep.2015.11.012

12. Sonneveld MJ, Brouwer WP, Chan HLY, et al. Optimisation of the use of APRI and FIB-4 to rule out cirrhosis in patients with chronic hepatitis B: results from the SONIC-B study. Lancet Gastroenterology \& Hepatology. Jul 2019;4(7):538-544. doi:10.1016/s2468-1253(19)30087-1

13. Wai C-T, Cheng CL, Wee A, et al. Non-invasive models for predicting histology in patients with chronic hepatitis B. Liver International. Aug 2006;26(6):666-672. doi:10.1111/j.1478-3231.2006.01287.x

14. Kong QF, Mei GT, Yang YX, Li Y, Shao YF, Miu XQ. Entecavir combined with Anluohuaxian pills in the treatment of 56 patients with hepatitis B cirrosis: a 5-year analysis. Chinese Journal of Intergrated Traditional and Western Medicine on Liver Diseases. 2020;30(06):506-508.

15. Lu W, Gao YH, Wang ZZ, et al. Effects of Anluohuaxianwan on transforming growth factor- $\beta 1$ and related signaling pathways in rats with carbon tetrachloride-induced liver fibrosis. Chin $\mathrm{J}$ Hepatol. $2017 ; 25(4): 257-262$. 
16. Wang L, Lu W, Gao Y-H, et al. Anluohuaxianwan alleviates carbon tetrachloride-induced hepatic fibrosis in rats through upregulation of peroxisome proliferator-activated receptor-Gamma and downregulation of nuclear factor-Kappa B/lкBa?; signaling pathway. Original Article. World Journal of Traditional Chinese Medicine. April 1, 2019 2019;5(2):95-103. doi:10.4103/wjtcm.wjtcm_16_19

17. She $\mathrm{H}$, Xiong $\mathrm{S}$, Hazra S, Tsukamoto $\mathrm{H}$. Adipogenic transcriptional regulation of hepatic stellate cells. J Biol Chem. Feb 11 2005;280(6):4959-67. doi:10.1074/jbc.M410078200

18. Rong G, Chen Y, Yu Z, et al. Synergistic Effect of Biejia-Ruangan on Fibrosis Regression in Patients with Chronic Hepatitis B Treated with Entecavir: A Multicenter, Randomized, Double-Blinded, PlaceboControlled Trial. J Infect Dis. May 21 2020;doi:10.1093/infdis/jiaa266

19. Huang C, Shen D, Sun S, et al. Effect of Fufang Biejia Ruangan Tablet on lowering biochemical and virological parameters of hepatic fibrosis in patients with chronic hepatitis B: Protocol for a systematic review and meta-analysis of randomized controlled trials and cohort studies. Medicine (Baltimore). Apr 2019;98(17):e15297. doi:10.1097/md.0000000000015297

20. Liu P, Hu YY, Liu C, et al. Multicenter clinical study on Fuzhenghuayu capsule against liver fibrosis due to chronic hepatitis B. World J Gastroenterol. May 21 2005;11(19):2892-9. doi:10.3748/wjg.v11.i19.2892

21. Gui HL, Zhao CQ, Wang Y, et al. Histological Outcome of Fuzheng Huayu plus Entecavir Combination Therapy in Chronic Hepatitis B Patients with Significant Liver Fibrosis. J Clin Trans/ Hepatol. Sep 28 2020;8(3):277-284. doi:10.14218/jcth.2020.00004

22. Liu HL, Lv J, Zhao ZM, et al. Fuzhenghuayu Decoction ameliorates hepatic fibrosis by attenuating experimental sinusoidal capillarization and liver angiogenesis. Sci Rep. Dec 10 2019;9(1):18719. doi:10.1038/s41598-019-54663-4

23. Poisson J, Lemoinne S, Boulanger $C$, et al. Liver sinusoidal endothelial cells: Physiology and role in liver diseases. J Hepatol. Jan 2017;66(1):212-227. doi:10.1016/j.jhep.2016.07.009

24. Loomba R, Liu J, Yang HI, et al. Synergistic effects of family history of hepatocellular carcinoma and hepatitis B virus infection on risk for incident hepatocellular carcinoma. Clin Gastroenterol Hepatol. Dec 2013;11(12):1636-45.e1-3. doi:10.1016/j.cgh.2013.04.043

25. Liver. EAftSot. EASL 2017 Clinical Practice Guidelines on the management of hepatitis $B$ virus infection. J Hepatol. Aug 2017;67(2):370-398. doi:10.1016/j.jhep.2017.03.021

26. McNaughton AL, D'Arienzo V, Ansari MA, et al. Insights From Deep Sequencing of the HBV GenomeUnique, Tiny, and Misunderstood. Gastroenterology. Jan 2019;156(2):384-399. doi:10.1053/j.gastro.2018.07.058

\section{Figures}




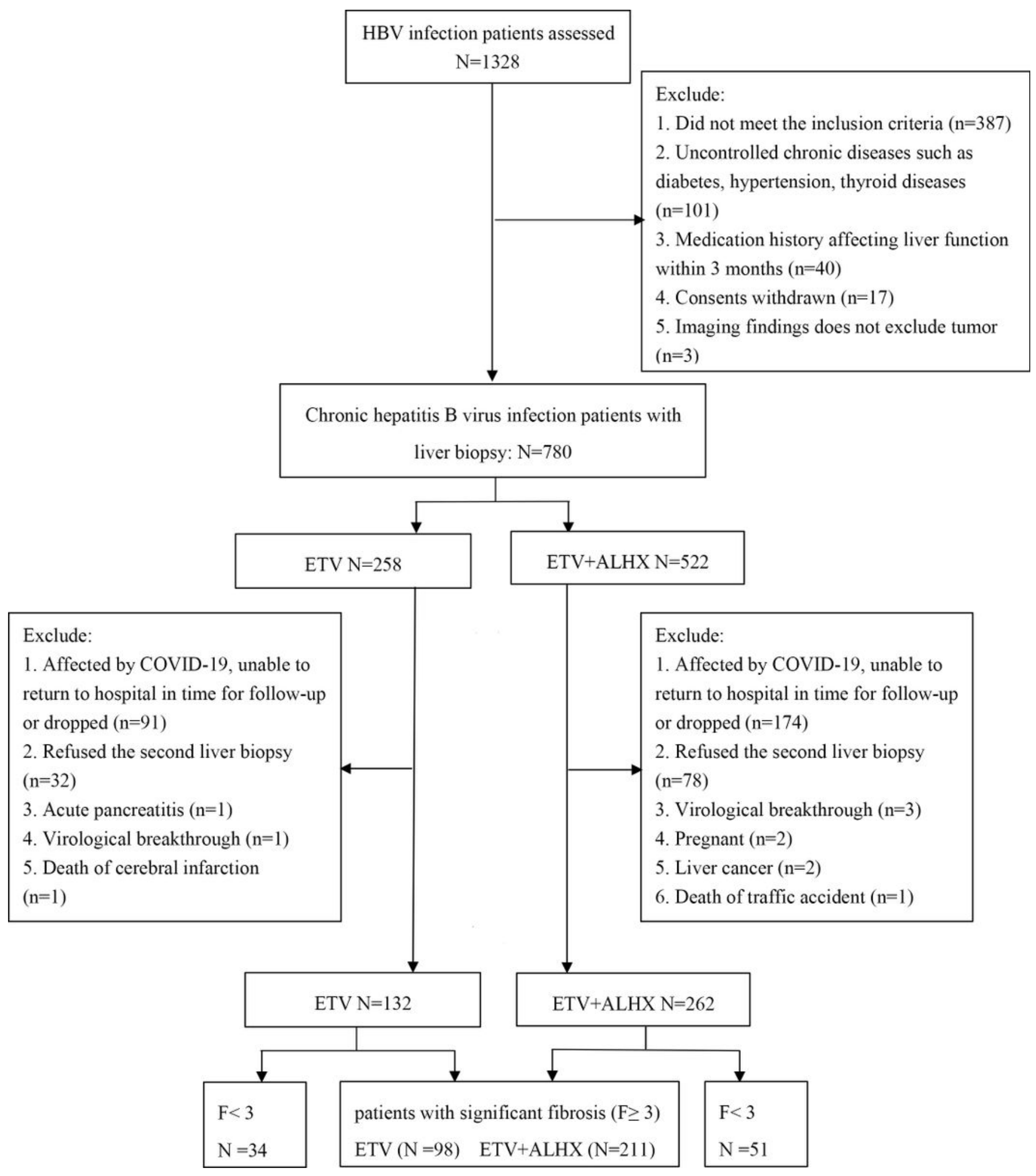

Figure 1

Flowchart of enrollment. 


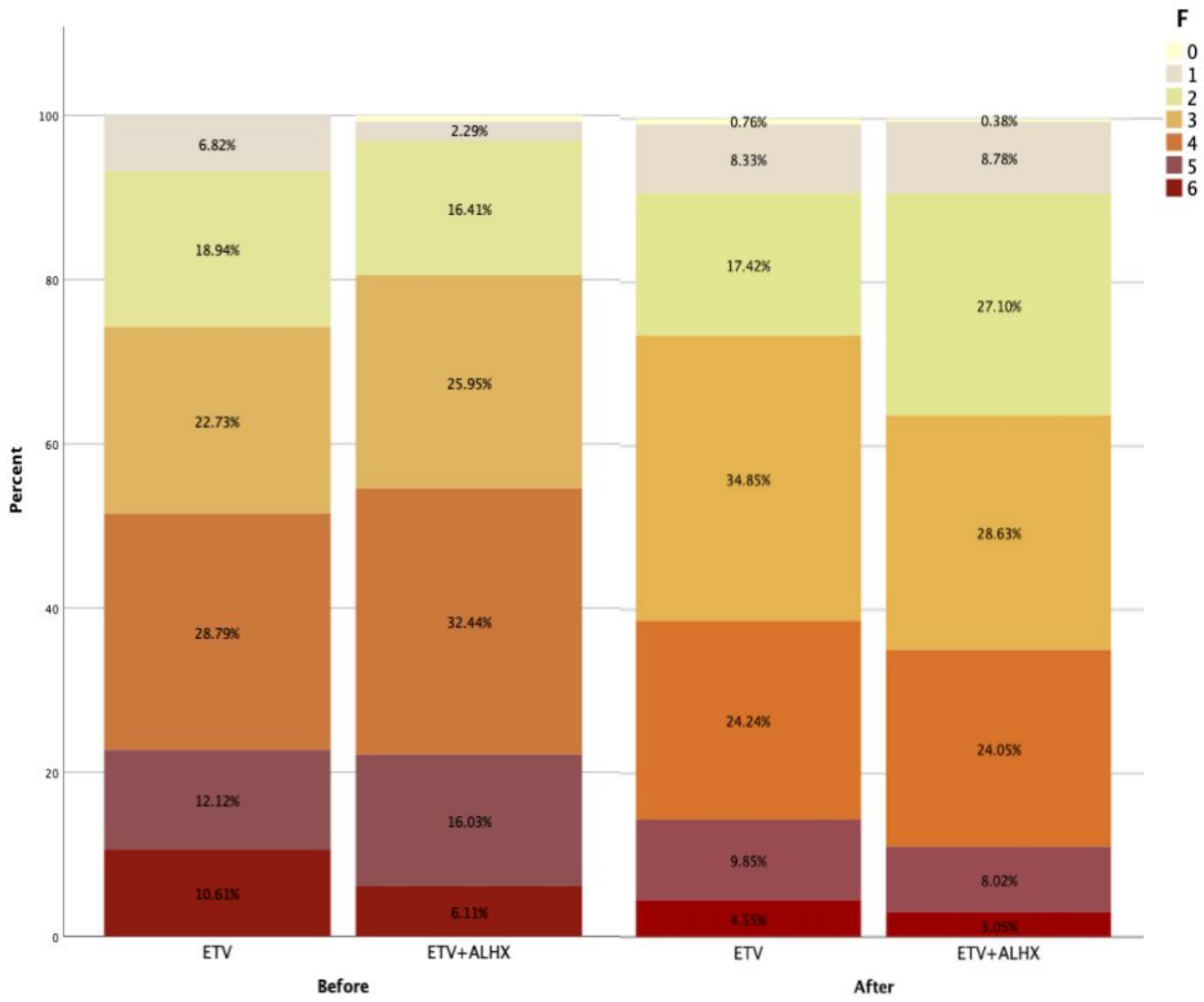

Figure 2

Distribution of fibrosis score in the two groups before and after $78 \mathrm{w}$ treatment. 

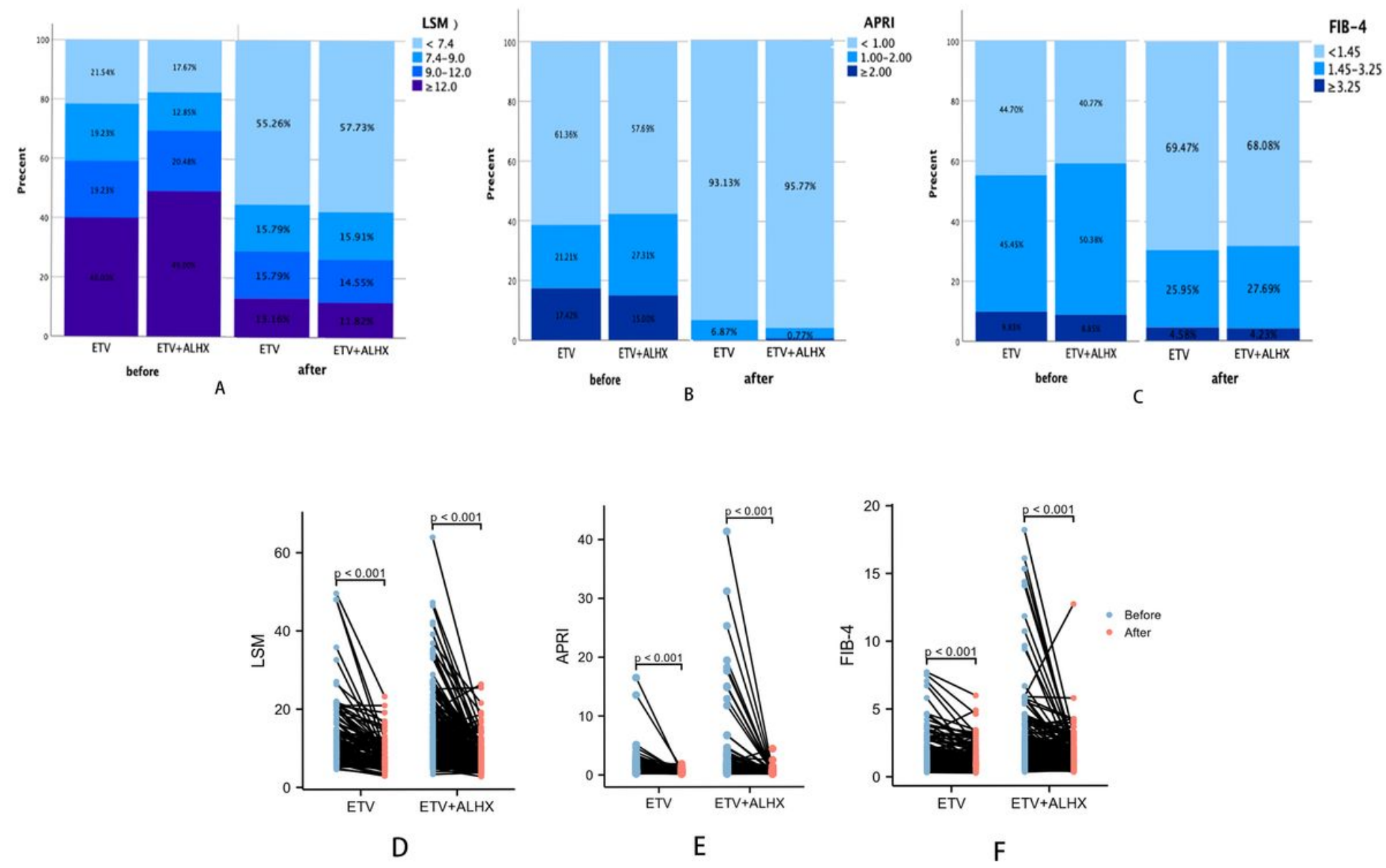

Figure 3

Distribution of LSM, APRI and FIB-4 in the two groups before and after treatment (A, B and C) and changes of them between the two groups before and after treatment (D, E and $F$ ).

\section{Supplementary Files}

This is a list of supplementary files associated with this preprint. Click to download.

- SupplementTables.docx 